\title{
Improved Bi-Directional Three-Phase Single-Relay Selection Technique for Cooperative Wireless Communications
}

\author{
Samer Alabed", Issam Maaz and Mohammad Al-Rabayah \\ College of Engineering and Technology, American University of the Middle East, Kuwait \\ *Corresponding Author: Samer Alabed. Email: samer.al-abed@aum.edu.kw \\ Received: 23 April 2021; Accepted: 24 May 2021
}

\begin{abstract}
Single-relay selection techniques based on the max-min criterion can achieve the highest bit error rate (BER) performance with full diversity gain as compared to the state-of-the-art single-relay selection techniques. Therefore, in this work, we propose a modified max-min criterion by considering the differences among the close value channels of all relays while selecting the best relay node. The proposed criterion not only enjoys full diversity gain but also offers a significant improvement in the achievable coding gain as compared to the conventional one. Basically, in this article, an improved bi-directional three-phase single-relay selection technique using the decodeand-forward protocol for wireless cooperative communication networks that enhances the overall network performance in terms of BER is proposed and its performance is proved analytically and through Monte-Carlo simulations. More specifically, the proposed criterion is first used to select the best relaynode. After that the selected relay-node forwards the information symbols of the communicating terminals after performing a digital network coding to minimize power consumptions. In our simulations, we show that our proposed technique outperforms the best-known single relay selection techniques. Furthermore, we prove that the BER results obtained from our conducted simulations perfectly match those obtained from the theoretical analysis.
\end{abstract}

Keywords: Performance analysis; cooperative diversity schemes; digital network coding; relay selection schemes

\section{Introduction}

In wireless communication systems, the overall performance and the data rate are highly affected by the multi-user interference and channel impairments such as the time varying fading caused by multi-path propagation [1-9]. Those channel impairments can be mitigated by using distributed beamforming techniques [2-6], distributed space-time-coding techniques [5-9], and relay-node selection techniques [10-22]. Such techniques allow the destination node to receive many copies of the same data signal through different paths and with different amplitudes and phases, which will be combined in a way to improve the overall system performance in terms of the whole network achieved data and bit error rate (BER). In cooperative communication

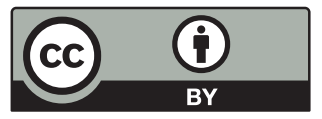

This work is licensed under a Creative Commons Attribution 4.0 International License, which permits unrestricted use, distribution, and reproduction in any medium, provided the original work is properly cited. 
networks, relay-nodes will jointly process the received data messages before broadcasting them using their antennas toward the final receiving entities, which will combine all the received copies from different paths to improve the spatial diversity gain. Such techniques are called the multi-antenna diversity techniques that incorporate the use of one-way or two-way relaying schemes [1-7].

In some scenarios, perfect channel state information (CSI) is assumed to be available at all nodes with slow fading channels [2,3,10,23]. In other scenarios, partial CSI is assumed to be available at the receiving antennas only [8,24,25]. Recently, the need for CSI data becomes unnecessary at both the sender and the receiver sides due to the usage of non-coherent and differential strategies [4-9,25-27]. Even though those techniques with no CSI data eliminate the overhead involved in channel estimation, they suffer from a low BER and spectral efficiency, as well as, high latency and decoding complexity.

In bi-directional communication scenarios, two entities send and receive messages through many relay-nodes [2-7]. Bi-directional relaying schemes can be classified based on the number of time slots required for exchanging the messages between the participating entities into three groups: two-phase [9,25,26], three-phase [13,25,26], and four-phase [2-6] protocols. The spectral efficiency of the four-phase protocols can be improved by reducing the number of transmission periods from four into three slots (three-phases) or two slots (two-phases). This reduction in the needed number of transmission slots has improved the overall performance of the two and three phase schemes compared to the four-phase ones [7,25]. In addition, relay-nodes transmit the received signals on orthogonal channels or encode them using either orthogonal or non-orthogonal coding. Full diversity gain with low decoding complexity is achieved by using orthogonal coding techniques such as space time block coding (STBC) $[8,25]$ compared to the high decoding complexity resulted from using non-orthogonal coding techniques. To obtain a full spatial diversity gain with low decoding complexity in non-orthogonal relay-node networks, relay-node selection techniques can be adopted $[13,21,22]$. Therefore, many single- and dual-relay selection strategies have been recently proposed.

Note that the $5 \mathrm{G}$ deployments adopt quite a few numbers of small cells with low power levels. Therefore, considering interference is crucially important, different kinds of detectors, cognitive radio methods, and cross-layer designs can be used to help improving the performance such as sigmoid function detectors and optimized target packet error rate [28-31].

In single-relay selection techniques, a single relay-node is selected among a group of relays to maximize either the signal to noise ratio (SNR) or the achievable data rate [10-13] where the best known single-relay selection algorithm, which is the optimal one, is the max-min algorithm proposed in [11]. However, this algorithm is applicable for single relay selection techniques. In [10, $13,21,22]$, dual-relay selection techniques have been suggested. However, two relays should be used in the network in the case of dual-relay selection techniques instead of just one in the case of single-relay section techniques. Even though some promising algorithms are recently proposed to be used in dual-relay selection techniques [10,13,21,22], e.g., in [10], the authors suggested to use the double-max criterion to select the best two relays.

The state-of-the-art articles investigating the single-relay selection techniques consider only the weakest or the strongest channels to select the best one or two relay nodes [10-13], e.g., they choose the best relay among all available relay nodes that owns the strongest forward or backward channel or they select two relays where one of them has the strongest forward channel and the other owns the strongest backward channel. Therefore, the motivation of this work is that the 
current techniques are not considering the differences among the close value channels while this can significantly improve the performance of the relaying techniques. For instance, the authors of [11-13] are selecting two relays using a certain criterion like the max-min criterion, which is considered as the optimal technique for selecting only a single relay. In this paper, the proposed algorithm, explained in Section 2, verifies whether the selected relays using the max-min criterion are the best ones before using them. Therefore, in this paper, we introduce a new two-relay selection strategy based on STC using the three-phase relaying protocol. In the suggested strategy, two relays out of $\mathrm{N}$ available relay-nodes are chosen in a way to optimize the system performance in terms of BER. Additional improvement is achieved by performing a digital network-coding scheme at the selected relay-nodes, which reduces the power consumption by merging the symbols of the communicating terminals in a single symbol with the same constellation.

Although single-relay selection techniques based on the max-min criterion enjoys a full diversity gain, there is still a margin to improve its coding gain [10-13,27]. Note that the diversity gain is related to the slope of the BER curve at very high SNR values while the coding gain is related to the horizontal shift of the BER curve to the left or the right, e.g., by doubling the power, the BER curve will be shifted $3 \mathrm{~dB}$ to the left without any change to the slope of the BER curve. Therefore, these techniques can achieve the highest diversity gain, however their BER curve can be enhanced by improving the coding gain. Thus, in our paper, we modify the max-min criterion by considering the differences among the nodes with close value channels. By applying this, we can improve the coding gain without losing any diversity gain. Through our simulations and analytical results, we have proved that our proposed technique outperforms the best-known state-of-the-art single-relay selection techniques [10-13]. In addition, we show that the BER results obtained from the conducted simulations match the ones obtained from the theoretical analysis. This paper is summarized as follows: Section 2 discusses the material and methods describing the system model. Section 3 explains the theory and calculation of the BER. Sections 4 and 5 demonstrate the achieved results and the related discussion of results. The conclusion is drawn in Section 6 .

\section{System Model}

In this section, we discuss our system model, in which we assume having a network composed of two single-antenna peripheral entities $T_{1}$ and $T_{2}$ that are separated by a distance exceeding their own transmission range, but want to exchange data messages between them. Therefore, a possible way to complete this needed communication between $T_{1}$ and $T_{2}$ is by selecting a single relay-node located between them. This intermediate relay-node is selected from a group of $\mathcal{R}$ single-antenna relay-nodes $\left(\boldsymbol{\mathcal { R }}_{1}, \ldots, \boldsymbol{\mathcal { R }}_{\mathrm{R}}\right)$, that are located between $\mathrm{T}_{1}$ and $\mathrm{T}_{2}$ as shown in Fig. 1.

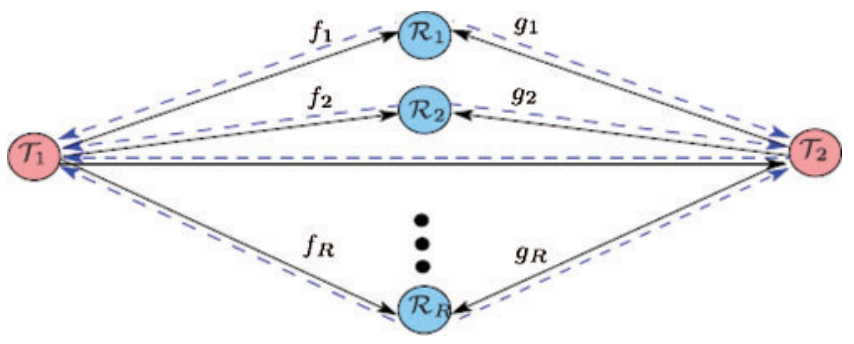

Figure 1: Two-way relay network 
The two communicating peripheral entities $T_{1}$ and $T_{2}$ and all the intermediate relay-nodes $\mathcal{R}_{1}, \ldots, \mathcal{R}_{\mathrm{R}}$ have a limited average transmit powers $\mathrm{P}_{\mathrm{T}_{1}}, \mathrm{P}_{\mathrm{T}_{2}}, \mathrm{P}_{\mathcal{R}_{1}}, \ldots, \mathrm{P}_{\mathcal{R}_{\mathrm{R}}}$, respectively. In this work, we consider a rich scattering environment without any line-of-sight components between the transmitter and the receiver; therefore, the channels follow a Rayleigh distribution. Also, we assume that the communication channels, from the intermediate relay-nodes to the peripheral entities and vice versa, are reciprocal for transmissions, and we denote them as $\mathrm{f}_{\mathrm{r}}$ (from $\mathrm{T}_{1}$ to $\boldsymbol{\mathcal { R }}_{\mathrm{r}}$ ) and $\mathrm{g}_{\mathrm{r}}$ (from $\mathrm{T}_{2}$ to $\boldsymbol{\mathcal { R }}_{\mathrm{r}}$ ). Considering the example shown in Fig. 2 where the network channel values are given. Based on the max-min selection criteria, $\mathcal{R}_{1}$ will be selected as the optimal relay-node as it has the maximum value link of 5 among the group of minimum value links (5, 4.9, and 3). However, we can clearly see that $\boldsymbol{\mathcal { R }}_{2}$ will probably have a much better performance compared to $\mathcal{R}_{1}$, as the difference between the minimum value links between $\boldsymbol{\mathcal { R }}_{1}$ and $\boldsymbol{\mathcal { R }}_{2}$ is only $2 \%((5-4.9) / 5) \times 100 \%$, while the maximum value link for $\mathcal{R}_{2}$ is 20 compared to 6 for $\mathcal{R}_{1}$, which is about $70 \%$ difference. Therefore, our proposed technique selects the best two relay-nodes based on the max-min criteria, and then it will check the difference between their minimum value links. If this difference is above a certain threshold, then the first selected relay-node will be chosen as the optimal relay-node.

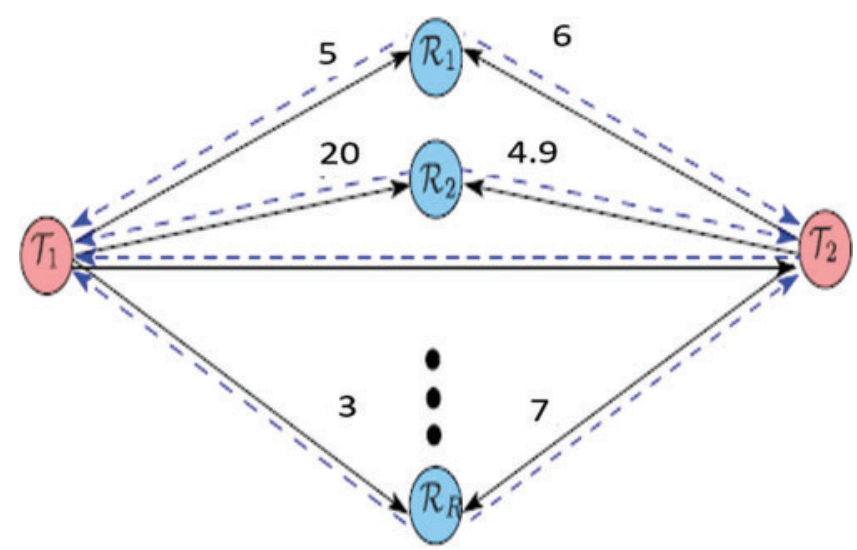

Figure 2: Two-way relay network example

On the other hand, if this difference is below the selected threshold, then we need to check the expected performance of both selected relay-nodes, and then we select the one with the highest performance. A detailed flow-chart that clarifies the operation of the proposed technique is shown in Fig. 3.

We assume here that all the relay and terminal-nodes have limited average transmit powers denoted as $\mathrm{P}_{\mathrm{T}_{\mathrm{j}}}, \mathrm{j}=1,2$ for terminal nodes and $\mathrm{P}_{\mathcal{R}_{\mathrm{i}}}, \mathrm{i} \in[1: \mathrm{N}]$ for relay-nodes. Furthermore, all intermediate communication channels between terminal and relay-nodes are assumed to be reciprocal for transmissions, and are denoted as $f_{i}$ from $T_{1}$ to relay-node $i$ and $g_{i}$ from $T_{2}$ to relay-node i. Furthermore, in our scenario we consider that the vision between the transmitters and receivers is obstructed thus only the non-line of sight scenarios are taken into consideration. As a result, $\mathrm{f}_{\mathrm{i}}$ and $\mathrm{g}_{\mathrm{i}}$ follow the Rayleigh distribution.

Finally, we use the following notations $|$.$| , L.J, (.)*, [a]_{i}, \bmod (a, b)$, and $E($.$) to denote$ the absolute value, the floor function which rounds toward zero, the complex conjugate, the ith element of the vector $a$, the remainder of the division of a by $b$, and the statistical expectation, 
respectively. In addition, the following abbreviations are used in this paper, BER: Bit error rate; bpcu: Bit per channel use; BPSK: Binary phase shift key; CSI: Channel state information; DF: Decode and forward; MGF: Moment generation function; ML: Maximum likelihood, SNR: Signal to noise ratio; STC: Space time coding; and QAM: Quadrature amplitude modulation, XOR: Exclusive OR, MA: Modular arithmetic.

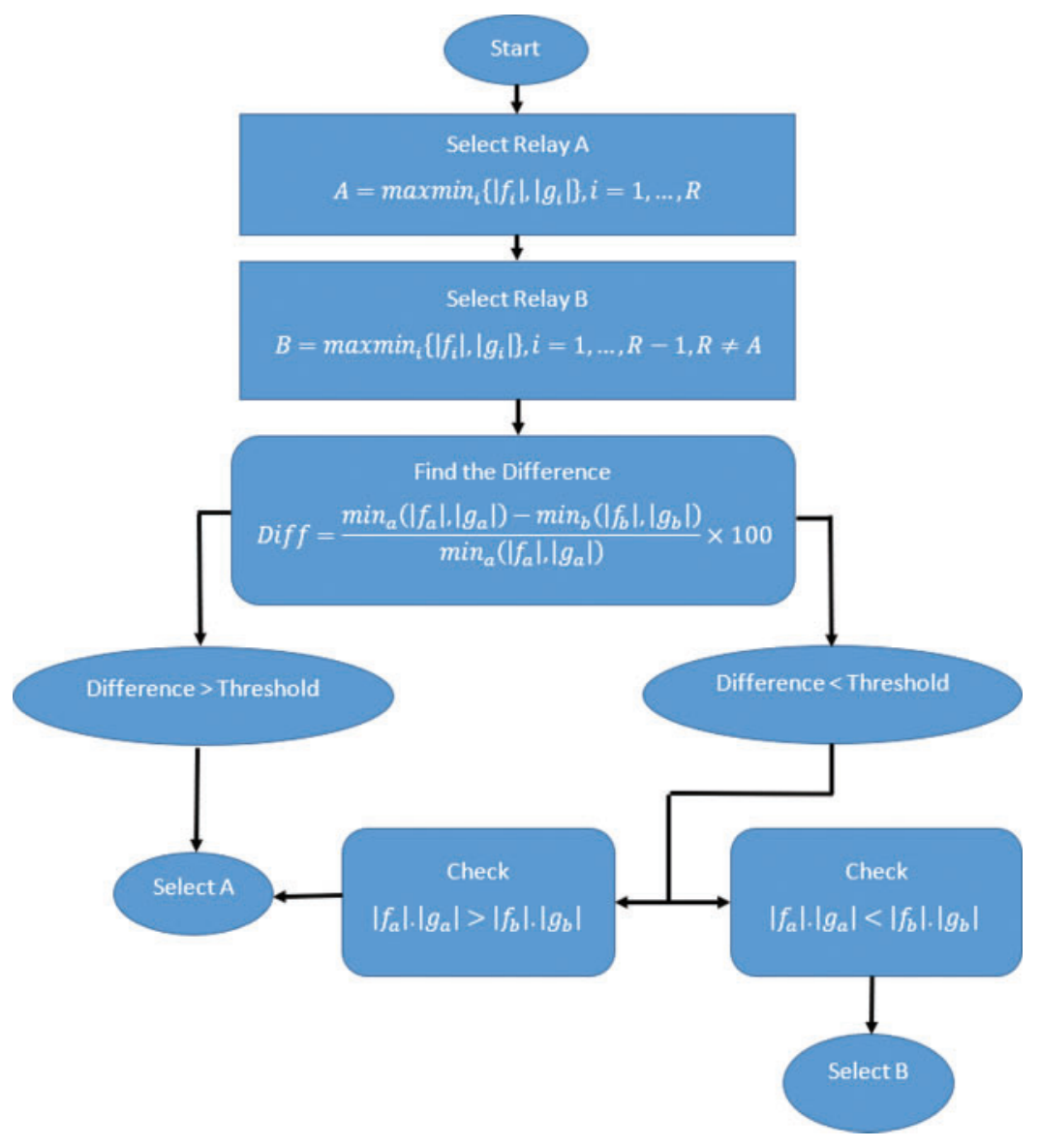

Figure 3: Flowchart of the proposed relay-node selection technique

\subsection{The Modified Max-Min Criterion}

In this proposed technique, one intermediate relay-node will be chosen from the available $\mathrm{R}$ relay-nodes using the modified max-min selection criterion. The used selection technique consists of three steps. In the first step, the best two relay-nodes $\left(\mathcal{R}_{\mathrm{a}}\right)$ and $\left(\boldsymbol{\mathcal { R }}_{\mathrm{b}}\right)$ will be selected among the available $\mathrm{R}$ intermediate relay-nodes based on the max-min selection criterion, in a way that the selected relay-nodes are the best ones that maximize the minimum value channels between the chosen relay-nodes and the two peripheral entities $T_{1}$ and $T_{2}$. The second step consists of defining a new parameter, called Diff, which calculates the difference between the minimum link values of the chosen relay-nodes in step 1. In step three, the best relay-node will be chosen based on a comparison between the calculated Diff value and the selected threshold. The threshold value is 
selected in a way to maximize the network performance through minimizing the BER, as will be shown in the next Section, i.e., Section 3.

In the first step, the first optimal single relay-node $\mathcal{R}_{\mathrm{a}}$ is chosen among the $\mathrm{R}$ available intermediate relay-nodes according to the max-min selection technique based on the below equation:

$\mathcal{R}_{a}=\arg _{a} \max \min \left(\left|f_{a}\right|,\left|g_{a}\right|\right) ; \quad a \in[1: R]$

Then, the second-best relay-node $\boldsymbol{\mathcal { R }}_{\mathrm{b}}$ is chosen from the remaining $(\mathrm{R}-1)$ relay-nodes based on the max-min selection technique as shown in the below equation:

$\boldsymbol{\mathcal { R }}_{b}=\arg _{b} \max \min \left(\left|f_{b}\right|,\left|g_{b}\right|\right) ; \quad b \in[1: R-1] ; \quad$ and $\quad b \neq a$

As it is observed from (1) and (2), the proposed technique chooses two relay-nodes ( $\boldsymbol{\mathcal { R }}_{\mathrm{a}}$ and $\mathcal{R}_{\mathrm{b}}$ ) according to the max-min relay-node selection criteria. The third step of the proposed technique consists of calculating the difference in the quality of links between the sender and the first selected relay-node $\boldsymbol{\mathcal { R }}_{\mathrm{a}}$ and the receiver, with the link between the sender and the second selected relay-node $\mathcal{R}_{\mathrm{b}}$ and the receiver according to the following equation:

$\operatorname{Diff}=\frac{\min \left(\left|f_{a}\right|,\left|g_{a}\right|\right)-\min \left(\left|f_{b}\right|,\left|g_{b}\right|\right)}{\min \left(\left|f_{a}\right|,\left|g_{a}\right|\right)} \times 100 \%$

This Diff parameter allows us to select the best relay-node, i.e., $\boldsymbol{\mathcal { R }}_{\mathrm{a}}$ or $\boldsymbol{\mathcal { R }}_{\mathrm{b}}$, based on comparing the obtained Diff value with the selected threshold value, i.e., $10 \%$. In this step, there are two possible findings as shown below:

$\left\{\begin{array}{l}\text { Diff }>\text { Threshold } \\ \text { Diff }<\text { Threshold }\end{array}\right.$

If the calculated Diff $>$ Threshold, then the best selected relay-node is $\mathcal{R}_{\mathrm{a}}$ since it has the best links between the sender and the receiver, and the difference between its links quality to the node with the second best links quality is greater than the specified Threshold, which offers the best performance. For Diff $<$ Threshold, the selected relay-node $\left(\boldsymbol{\mathcal { R }}_{\mathrm{S}}\right)$ will be chosen as follows:

$\mathcal{R}_{s}= \begin{cases}\mathcal{R}_{b} & \left|f_{b}\right| \cdot\left|g_{b}\right|>\left|f_{a}\right| \cdot\left|g_{a}\right| \\ \mathcal{R}_{a} & \left|f_{b}\right| \cdot\left|g_{b}\right|<\left|f_{a}\right| \cdot\left|g_{a}\right|\end{cases}$

This means that relay-node $\boldsymbol{\mathcal { R }}_{\mathrm{b}}$ will be selected only if there is a slight difference between its minimum value link and that of relay-node $\boldsymbol{\mathcal { R }}_{\mathrm{a}}$, and its overall expected performance is higher than the expected performance from relay-node $\mathcal{R}_{\mathrm{a}}$.

\subsection{The Proposed Three-Phase Single Relay Selection Technique}

In this part, three-phase decode and forward communication protocol will be performed. The first peripheral entity, $\mathrm{T}_{1}$, will transmit its data message $\mathrm{s}_{\mathrm{T}_{1}}$ in the first time slot, then, the second peripheral entity, $\mathrm{T}_{2}$, will transmit its data message, $\mathrm{s}_{2}$, in the second time slot, such that the sth relay-node $\mathcal{R}$ s receives the following messages:

$y_{\mathcal{R}_{1}, s}=\sqrt{P_{T_{1}}} f_{s} s_{T_{1}}+n_{\mathcal{R}_{1}, s}$ 
$y_{\mathcal{R}_{2, s}}=\sqrt{P_{T_{2}}} g_{s} s_{T_{2}}+n_{\mathcal{R}_{2}, s}$

where $\mathrm{n}_{\mathcal{R}_{1}, \mathrm{~s}}$ and $\mathrm{n}_{\mathcal{R}_{2} \text {,s }}$ denote the noise signals received at the sth relay-node $\mathcal{R} \mathrm{s}$ in the first and the second time slots. Now, the sth relay-node $\mathcal{R}_{\mathrm{s}}$ decodes the data messages received from the first and the second entities using the maximum likelihood (ML) decoder as shown below:

$\tilde{s}_{T_{1, s}}=\arg _{s} \min \left|y_{\mathcal{R}_{1}, s}-\sqrt{P_{T_{1}}} f_{s} s_{T_{1}}\right|$

$\tilde{s}_{T_{2, s}}=\arg _{s} \min \left|y_{\mathcal{R}_{2}, s}-\sqrt{P_{T_{2}}} g_{s} s_{T_{2}}\right|$

Note here that the relay-node decoding complexity is very low, as a symbol-wise decoder, which enjoys a linear decoding complexity is being used to detect the received data messages. At this stage, the rth relay node $\mathcal{R} \mathrm{r}$ will combine the two received messages, $\tilde{\mathrm{s}}_{\mathrm{T}, \mathrm{r}}$ and $\tilde{\mathrm{s}}_{\mathrm{T}, \mathrm{r}}$, to form a single message as follows:

$s_{\mathcal{R}, s}=\mathcal{F}\left(\tilde{s}_{T_{1, s}}, \tilde{s}_{T_{2, s}}\right)$

where $\mathcal{F}(.,$.$) is a function used to combine the decoded symbols. Recently, many functions have$ been proposed, e.g., modular arithmetic (MA) function [25]. Note that $\mathrm{s}_{\mathcal{R}, \mathrm{s}} \in \mathrm{S}_{\mathcal{R}}, \tilde{\mathrm{s}}_{\mathrm{T}_{1, \mathrm{~s}}} \in \mathrm{S}_{\mathrm{T}_{1}}$ and $\tilde{\mathrm{s}}_{\mathrm{T}_{2, \mathrm{~s}}} \in \mathrm{S}_{\mathrm{T}_{2}}$ where $\mathrm{S}_{\mathcal{R}}, \mathrm{S}_{\mathrm{T}_{1}}$ and $\mathrm{S}_{\mathrm{T}_{2}}$ denote the constellation of the information symbols transmitted from the selected relay node, the first terminal, and the second terminal, respectively, and $\left|\mathrm{S}_{\mathcal{R}}\right|=$ $\max \left\{\left|\mathrm{S}_{\mathrm{T}_{1}}\right|,\left|\mathrm{S}_{\mathrm{T}_{2}}\right|\right\}$. Let us consider the $\mathrm{jth}$ entry $\mathrm{s}$ of a constellation $\mathrm{S}$ as $\mathrm{S}(\mathrm{j})$ where $\mathrm{j} \in\{0:|\mathrm{S}|-1\}$ and the inverse as $\mathrm{S}^{-1}(\mathrm{~s})=\mathrm{j}$. Let us define $\mathrm{k}_{\mathrm{T}_{1}}$ and $\mathrm{k}_{\mathrm{T}_{2}}$ as $\mathrm{S}_{\mathrm{T}_{1}}\left(\mathrm{k}_{\mathrm{T}_{1}}\right)=\tilde{\mathrm{s}}_{\mathrm{T}_{1, \mathrm{~s}}}$ and $\mathrm{S}_{\mathrm{T}_{2}}\left(\mathrm{k}_{\mathrm{T}_{2}}\right)=\tilde{\mathrm{s}}_{\mathrm{T}_{2, \mathrm{~s}}}$,

the MA function can be written as $\mathcal{F}_{\mathrm{m}}\left(\tilde{\mathrm{s}}_{\mathrm{T}_{1, \mathrm{~s}}}, \tilde{\mathrm{s}}_{\mathrm{T}_{2, \mathrm{~s}}}\right)=\mathrm{S}_{\mathcal{R}}\left(\bmod \left(\mathrm{S}_{\mathrm{T}_{1}}^{-1}\left(\tilde{\mathrm{s}}_{\mathrm{T}_{1, \mathrm{~s}}}\right)+\mathrm{S}_{\mathrm{T}_{2}}^{-1}\left(\tilde{\mathrm{s}}_{\mathrm{T}_{2, \mathrm{~s}}}\right),\left|\mathrm{S}_{\mathrm{R}}\right|\right)\right)=$ $\mathrm{S}_{\mathcal{R}}\left(\bmod \left(\mathrm{k}_{\mathrm{T}_{1}}+\mathrm{k}_{\mathrm{T}_{2}},\left|\mathrm{~S}_{\mathrm{R}}\right|\right)\right)$. Moreover, the exclusive OR (XOR) combination function suggested in [1] and the other combination function proposed in [3] can also be used to merge the information symbols. After that, the selected relay-node broadcasts the combined data message toward the two communicating peripheral entities in the third time slot. Thus, the second receiving peripheral entity receives the below message:

$y_{T_{2}}=\sqrt{P_{R_{a}}} g_{s} s_{\mathcal{R}, s}+n_{T_{2}}$

where $\mathrm{n}_{T_{2}}$ represents the noise signal at the second peripheral entity $\mathrm{T} 2$ received in the third time slot. Similarly, the ML decoder is performed to recover the received data at the second peripheral entity T2. It is worth mentioning that this peripheral entity can recover the data message using a symbol-by-symbol detector, which has a linear decoding complexity instead of applying the ML decoder, based on the knowledge of its own data message $\mathrm{s}_{\mathrm{T}_{1}}$ and by applying the inverse of the combination function used at the rth relay node $\mathcal{R}$ r.

\section{BER Performance Analysis}

In this section, we will discuss the mathematical model derivation of the BER of the proposed selection technique using binary phase shift keying (BPSK) modulation under the assumptions discussed in Section 2 earlier. Here, it is also assumed that all relay nodes are ideal as in $[2,9,12]$. In addition, all noise signals are assumed to be identically drawn from an independent Gaussian distributed random function with zero mean and $\sigma^{2} I_{T}$ covariance. Our proposed relaynode selection technique defined in (5) picks up the $\mathrm{s}_{\text {th }}$ relay-node having $\mathrm{P}_{\mathcal{R}_{\mathrm{s}}}=\mathrm{P}_{\mathcal{R}}$. Now, given that the SNR of the link between $\mathcal{R}_{\mathrm{r}}$ and $\mathrm{T}_{1}$ is denoted by $\gamma_{\mathrm{r}}^{\mathrm{T}_{1}}=\gamma\left|\mathrm{f}_{\mathrm{r}}\right|^{2}$, the SNR of the link 
between $\mathcal{R}_{\mathrm{r}}$ and $\mathrm{T}_{2}$ is denoted by $\gamma_{\mathrm{r}}^{\mathrm{T}_{2}}=\gamma\left|\mathrm{g}_{\mathrm{r}}\right|^{2}$, and $\gamma=\mathrm{P}_{\mathcal{R}} / \sigma^{2}$ is the mean SNR at the $\mathrm{r}_{\text {th }}$ relay $\mathcal{R}_{\mathrm{r}}$, let us rearrange $\gamma_{\mathrm{r}}^{\mathrm{T}_{\mathrm{t}}}, \mathrm{r} \in[1, \mathrm{R}]$ increasingly such that $\gamma_{1}^{\mathrm{T}_{\mathrm{t}}} \leq \gamma_{2}^{\mathrm{T}_{\mathrm{t}}} \leq \ldots \leq \gamma_{\mathrm{R}}^{\mathrm{T}_{\mathrm{t}}}$ and denote $\mathrm{w}_{1}^{\mathrm{T}_{1}}=\gamma_{1}^{\mathrm{T}_{\mathrm{t}}}$ and $\mathrm{w}_{1}^{\mathrm{T}_{1}}=\gamma_{1}^{\mathrm{T}_{\mathrm{t}}}-\gamma_{(1-1)}^{\mathrm{T}_{\mathrm{t}}}$ for $1 \in[2, \mathrm{R}]$. Note here that those independent factors, $\mathrm{w}_{1}$ for $1 \in[1, \mathrm{R}]$, are derived using a probability distribution function, expressed as [13,22,27]:

$f_{w_{l}}\left(w_{l}\right)=\frac{R-l-1}{\gamma} \exp \left(-\frac{R-l-1}{\gamma} w_{l}\right)$

In the proposed technique, the $\mathrm{a}_{\text {th }}$ relay-node $\boldsymbol{\mathcal { R }}_{\mathrm{a}}$ is selected among the $\mathrm{R}$ available relay-nodes based on the max-min criteria explained in (1) given that $\mathrm{f}_{\mathrm{a}}$ is the link between $\boldsymbol{\mathcal { R }}_{\mathrm{a}}$ and $\mathrm{T}_{1}$ with $\gamma_{\mathrm{u}_{1}}^{\mathrm{T}_{1}}=\gamma_{\left\lfloor\frac{\mathrm{R}}{2}\right\rfloor}^{\mathrm{T}_{1}}$, and $\mathrm{g}_{\mathrm{a}}$ is the link between $\mathcal{R}_{\mathrm{a}}$ and $\mathrm{T}_{2}$ with $\gamma_{\mathrm{u}_{2}}^{\mathrm{T}_{2}}$, where $\gamma_{\mathrm{u}_{2}}^{\mathrm{T}_{2}} \geq \gamma_{\left\lfloor\frac{\mathrm{R}}{2}\right\rfloor}^{\mathrm{T}_{1}}$ and $\mathrm{u}_{2}$ is any $\mathrm{u}_{\text {th }}$ greater than $\left\lfloor\frac{\mathrm{R}}{2}\right\rfloor$, i.e., $\mathrm{u}_{2}=\left\lfloor\frac{\mathrm{R}}{2}\right\rfloor+1, \ldots, \mathrm{R}$. After that, the $\mathrm{b}_{\text {th }}$ relay-node $\boldsymbol{\mathcal { R }}_{\mathrm{b}}$ is selected from the remaining $(\mathrm{R}-1)$ relay-nodes based on the max-min criteria explained in (2) given that $\mathrm{f}_{\mathrm{b}}$ is the link between $\mathcal{R}_{\mathrm{b}}$ and $\mathrm{T}_{1}$ with $\gamma_{\mathrm{u}_{3}}^{\mathrm{T}_{1}}=\gamma_{\left\lfloor\frac{\mathrm{R}-1}{2}\right\rfloor}^{\mathrm{T}_{1}}$, and $\mathrm{g}_{\mathrm{b}}$ is the link between $\mathcal{R}_{\mathrm{b}}$ and $\mathrm{T}_{2}$ with $\gamma_{\mathrm{u}_{4}}^{\mathrm{T}_{2}}$, where $\gamma_{\mathrm{u}_{4}}^{\mathrm{T}_{2}} \geq \gamma_{\left\lfloor\frac{\mathrm{R}-1}{2}\right\rfloor}^{\mathrm{T}_{1}}$ and $\mathrm{u}_{4}$ is any $\mathrm{u}_{\mathrm{th}}$ greater than $\left\lfloor\frac{(\mathrm{R}-1)}{2}\right\rfloor$, i.e., $\mathrm{u}_{4}=\left\lfloor\frac{(\mathrm{R}-1)}{2}\right\rfloor+1, \ldots, \mathrm{R}$. Assuming that $\gamma_{\mathrm{u}_{2}}^{\mathrm{T}_{2}} \geq \gamma_{\left\lfloor\frac{\mathrm{R}}{2}\right\rfloor}^{\mathrm{T}_{1}} \geq \gamma_{\left\lfloor\frac{\mathrm{R}-1}{2}\right\rfloor}^{\mathrm{T}_{1}}$, then, the average BER for the first round of the max-min relay-node selection technique, (selecting $\boldsymbol{\mathcal { R }}_{\mathrm{a}}$ ), can be expressed as:

$$
\begin{aligned}
P_{1}(\gamma) & =\frac{1}{2} E\left(\sum_{j=1}^{2} Q\left(\sqrt{2 \gamma_{u_{j}}^{T_{j}}}\right)\right)=\frac{1}{2} E\left(Q\left(\sqrt{2 \gamma_{u_{1}}^{T_{1}}}\right)+Q\left(\sqrt{2 \gamma_{u_{2}}^{T_{2}}}\right)\right) \\
& =\frac{1}{2} E\left(Q\left(\sqrt{2 \gamma_{\left\lfloor\frac{R}{2}\right\rfloor}^{T_{1}}}\right)+Q\left(\sqrt{2 \gamma_{u_{2}}^{T_{2}}}\right)\right)=\frac{1}{2}\left(L_{1}+L_{2}\right)
\end{aligned}
$$

where $\mathrm{u}_{2}>\left\lfloor\frac{\mathrm{R}}{2}\right\rfloor$.

$$
\begin{aligned}
L_{1} & =E\left(Q\left(\sqrt{2 \gamma_{\mid \frac{R}{2}}^{T_{1}}}\right)\right) \\
& =\frac{\sqrt{\gamma}}{2} \sum_{p=0}^{\left.\frac{R}{2}\right\rfloor}\left(\begin{array}{c}
\left\lfloor\frac{R}{2}\right\rfloor \\
P
\end{array}\right)(-1)^{P} \frac{1}{\sqrt{P+\gamma}} \\
L_{2} & =E\left(Q\left(\sqrt{2 \gamma_{u_{2}}^{T_{2}}}\right)\right)=\frac{\sqrt{\gamma}}{2} \sum_{p=0}^{u_{2}}\left(\begin{array}{c}
u_{2} \\
P
\end{array}\right)(-1)^{P} \frac{1}{\sqrt{P+\gamma}}
\end{aligned}
$$


Similarly, the average BER for the second round of the max-min relay-node selection technique, (selecting $\mathcal{R}_{\mathrm{b}}$ after excluding $\boldsymbol{\mathcal { R }}_{\mathrm{a}}$ ), can be expressed as:

$\mathrm{P}_{2}(\gamma)=\frac{1}{2} \mathrm{E}\left(\mathrm{Q}\left(\sqrt{2 \gamma_{\mathrm{u}_{3}}^{\mathrm{T}_{1}}}\right)+\mathrm{Q}\left(\sqrt{2 \gamma_{\mathrm{u}_{4}}^{\mathrm{T}_{2}}}\right)\right)=\frac{1}{2} \mathrm{E}\left(\mathrm{Q}\left(\sqrt{2 \gamma_{\left\lfloor\frac{(\mathrm{R}-1)}{2}\right\rfloor}^{\mathrm{T}_{1}}}\right)+\mathrm{Q}\left(\sqrt{2 \gamma_{\mathrm{u}_{4}}^{\mathrm{T}_{2}}}\right)\right)=\frac{1}{2}\left(\mathrm{~L}_{3}+\mathrm{L}_{4}\right)$

where $\mathrm{u}_{4}>\left\lfloor\frac{(\mathrm{R}-1)}{2}\right\rfloor$.

$$
\begin{aligned}
& L_{3}=E\left(Q\left(\sqrt{2 \gamma_{\left\lfloor\frac{(R-1)}{2}\right\rfloor}^{T_{1}}}\right)\right)=\frac{\sqrt{\gamma}}{2} \sum_{p=0}^{\left.\frac{(R-1)}{2}\right\rfloor}\left(\left\lfloor\frac{(R-1)}{2}\right\rfloor\right)(-1)^{P} \frac{1}{\sqrt{P+\gamma}} \\
& \mathrm{L}_{4}=\mathrm{E}\left(\mathrm{Q}\left(\sqrt{2 \gamma_{\mathrm{u}_{4}}^{\mathrm{T}_{2}}}\right)\right)=\frac{\sqrt{\gamma}}{2} \sum_{\mathrm{p}=0}^{\mathrm{u}_{4}}\left(\begin{array}{c}
\mathrm{u}_{4} \\
\mathrm{P}
\end{array}\right)(-1)^{\mathrm{P}} \frac{1}{\sqrt{\mathrm{P}+\gamma}}
\end{aligned}
$$

Therefore, the average BER for the proposed relay-node selection technique can be expressed as:

$P(\gamma)= \begin{cases}P_{2}(\gamma), & \text { Diff }>\text { Threshold, }\left|f_{b}\right|\left|g_{b}\right|>\left|f_{a}\right|\left|g_{a}\right| \\ P_{1}(\gamma), & \text { otherwise }\end{cases}$

From (19), it is clearly shown that the BER is a function of the number of relays, the transmitted power, and the constellation size.

\section{Results and Discussion}

In this section, we discuss the obtained results from both Monte Carlo simulations, as well as, analytical models discussed in Section 3. In the simulations, we have assumed a wireless relay network with single-antenna relay nodes and independent flat Rayleigh fading channels where the power is distributed among the two terminals and relays similarly as in [11-13], the number of Monte Carlo runs is $10^{10}$, the number of relays available in the network varies between two to seven, and the applied modulation technique is either BPSK or 4-QAM. For fair comparison of the BER performance of all techniques, the same total transmitted power and bit rate are used.

Fig. 4 shows the BER vs. the SNR results of a wireless cooperative network composed of two entities (T1 and T2) with no direct communication links available, and four relay nodes $(\mathrm{R}=4)$ where the 4-QAM constellation is used in this scenario. More specifically, this figure shows the performance results of a single [11] and dual [10] relay-node selection strategies using the three-phase relaying protocol with 4-QAM modulation in the presence of four relay-nodes under different threshold values (i.e., Threshold varies between 0 and 10).

The BER results for our proposed single-relay-node three-phase selection technique using 4-QAM modulation and with four relay-nodes available under different threshold values is depicted in Fig. 5. It is clearly shown that the BER decreases with the increase of the threshold value. The BER reaches its minimum for a threshold value equal to 4. After that, the BER 
increases with the increase of the threshold value. From (19), the BER is a function of the number of relays, the transmitted power, and the constellation size. By changing any of these factors, the optimal threshold value will change as well. Fig. 6 shows the BER vs. the SNR for a single and dual relay-node selection strategies using the three-phase relaying protocol with 4-QAM modulation in the presence of five relay-nodes $(R=5)$ under different threshold values i.e., Threshold varies between 0 and 10. Fig. 7 shows the relation between the BER performance of the relay-node network scenario discussed for Fig. 6 and the Threshold values. Similarly, Fig. 8 shows the BER vs. the SNR results for a single and dual relay-node selection strategies using the three-phase relaying protocol with 4-QAM modulation in the presence of seven relay-nodes $(\mathrm{R}=$ 7) under different threshold values i.e., Threshold varies between 0 and 10, and Fig. 9 shows the relation between the BER performance of the relay-node network scenario discussed for Fig. 8 under different threshold values.

Fig. 10 shows a comparison in BER performance vs. the SNR between the single-relay three-phase selection technique proposed in [11] and double-relay three-phase selection technique proposed in [10] with 8-QAM modulation using different number of relay nodes (i.e., $\mathrm{R}=2$, 4, 6). Finally, in Fig. 11, we show the theoretical and simulated BER performance vs. SNR for the proposed technique with binary phase shift keying (BPSK) modulation, using various numbers of relay-nodes, $\mathrm{R}=3,4$, and 7 , with a selected threshold value of four (Threshold $=4$ ). Hence, it can be concluded that the BER curve at a certain threshold value can be found using either (19) or Monte Carlo simulations and is changing based on many factors such as the used constellation size, the total transmitted power, and the number of relay nodes. Based on the conducted Monte Carlo simulations, we found that the optimal threshold value varies between 4 to 8 .

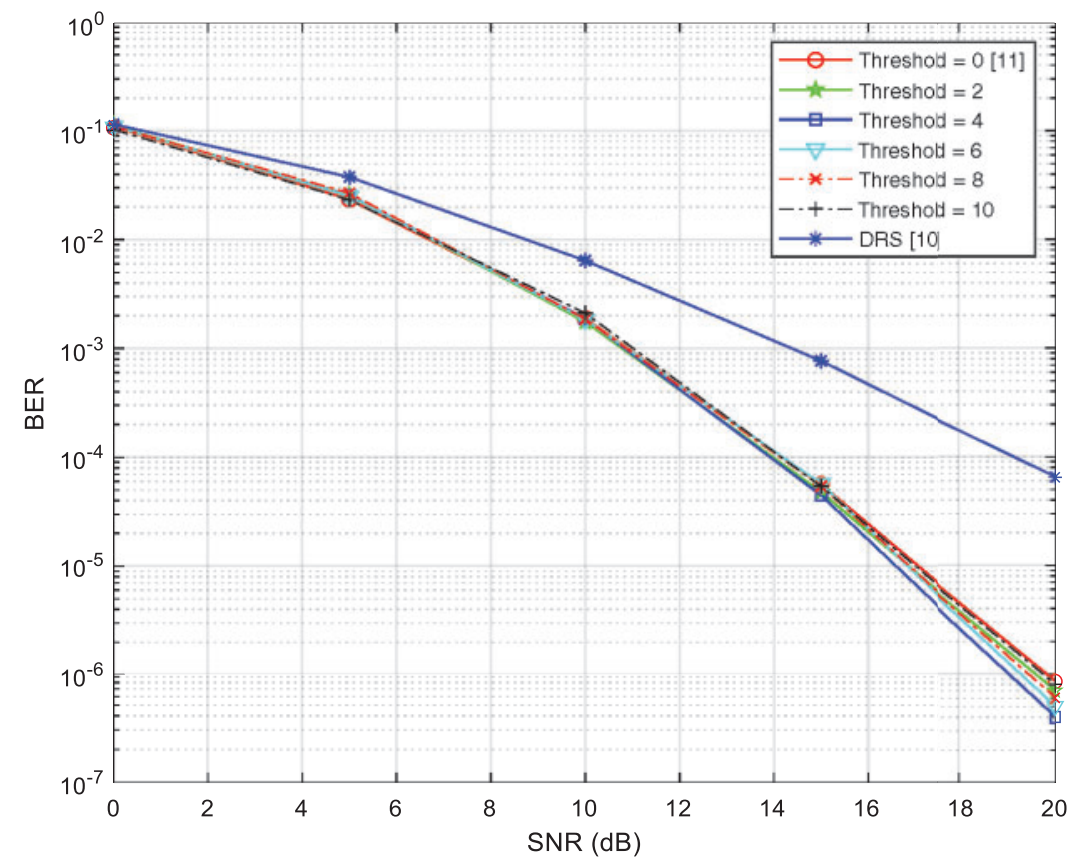

Figure 4: BER vs. SNR for single and dual relay selection strategies using the three-phase relaying protocol with 4-QAM modulation and $\mathrm{R}=4$ 


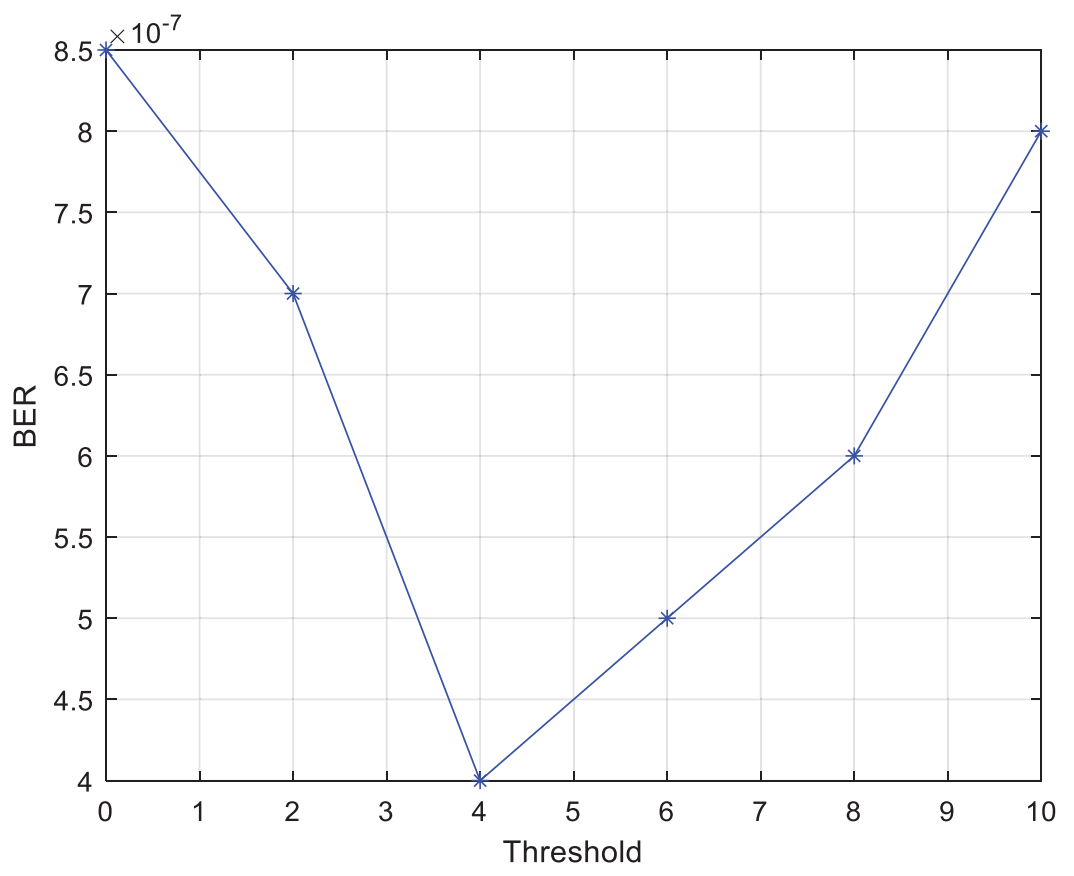

Figure 5: BER vs. Threshold value for single relay selection strategies using the three-phase relaying protocol with $4-\mathrm{QAM}$ modulation, $\mathrm{R}=4, \mathrm{SNR}=20 \mathrm{~dB}$

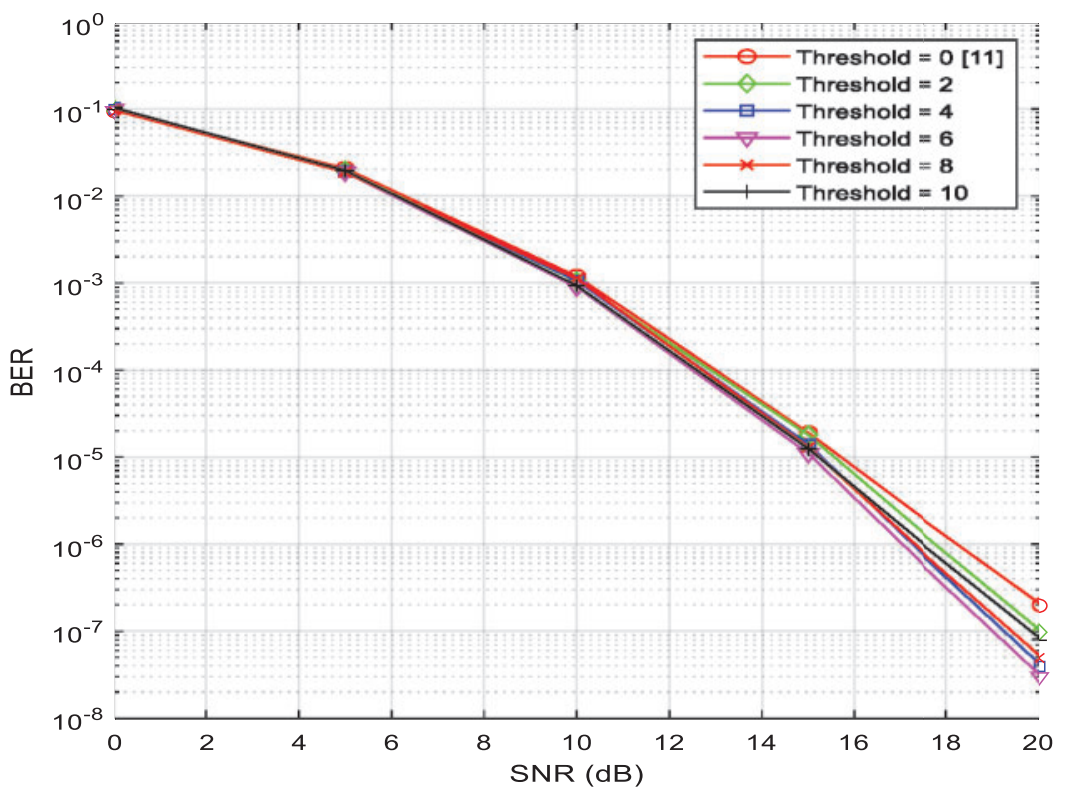

Figure 6: BER vs. SNR for single relay selection strategies using the three-phase relaying protocol with 4-QAM modulation and $\mathrm{R}=5$

From Fig. 4, it is clearly shown that our proposed three-phase single-relay-node selection technique under different threshold values outperforms both the three-phase double-relay-node 
selection technique proposed in [10] and the three-phase single-relay-node selection technique proposed in [11]. Noting that our proposed technique using a threshold value of zero. It is worth mentioning that the proposed technique which implements a three-phase relaying protocol outperforms the current state-of-the-art strategies that perform the same relaying protocol. Fig. 5 shows the relation between the BER performance of the relay-node network scenario discussed for Fig. 4 and the threshold values. It is observed that the BER will improve while increasing the threshold value, until the best performance is achieved at threshold value of four (Threshold $=$ 4), then the performance will degrade for threshold values smaller than four.

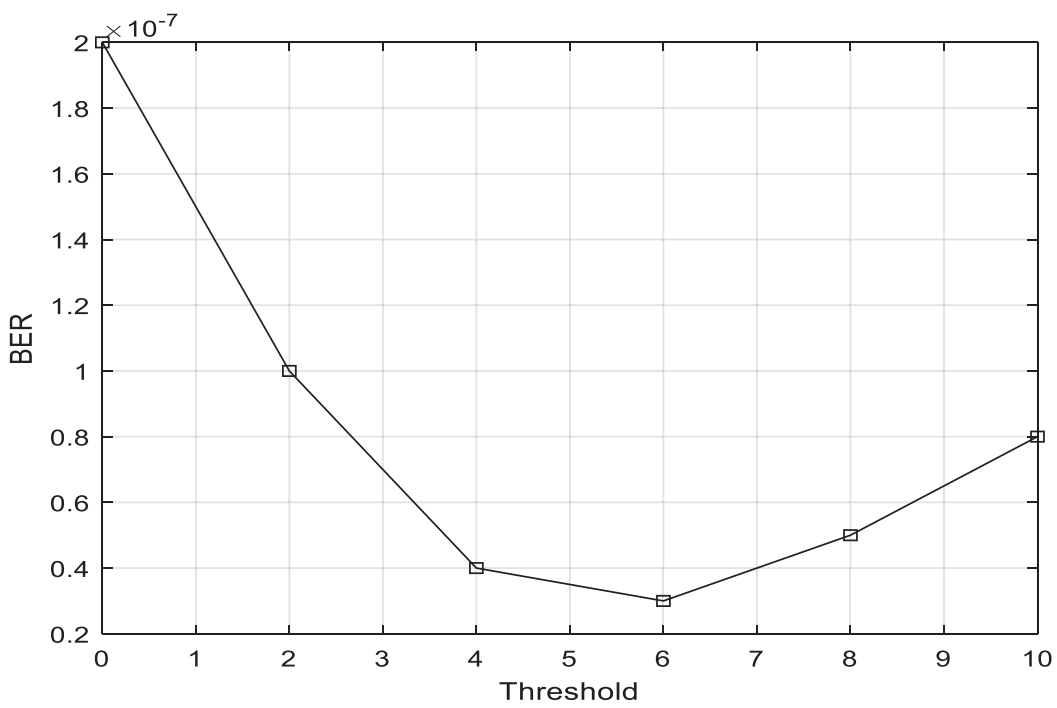

Figure 7: BER vs. Threshold value for single relay selection strategies using the three-phase relaying protocol with $4-\mathrm{QAM}$ modulation, $\mathcal{R}=5, \mathrm{SNR}=20 \mathrm{~dB}$

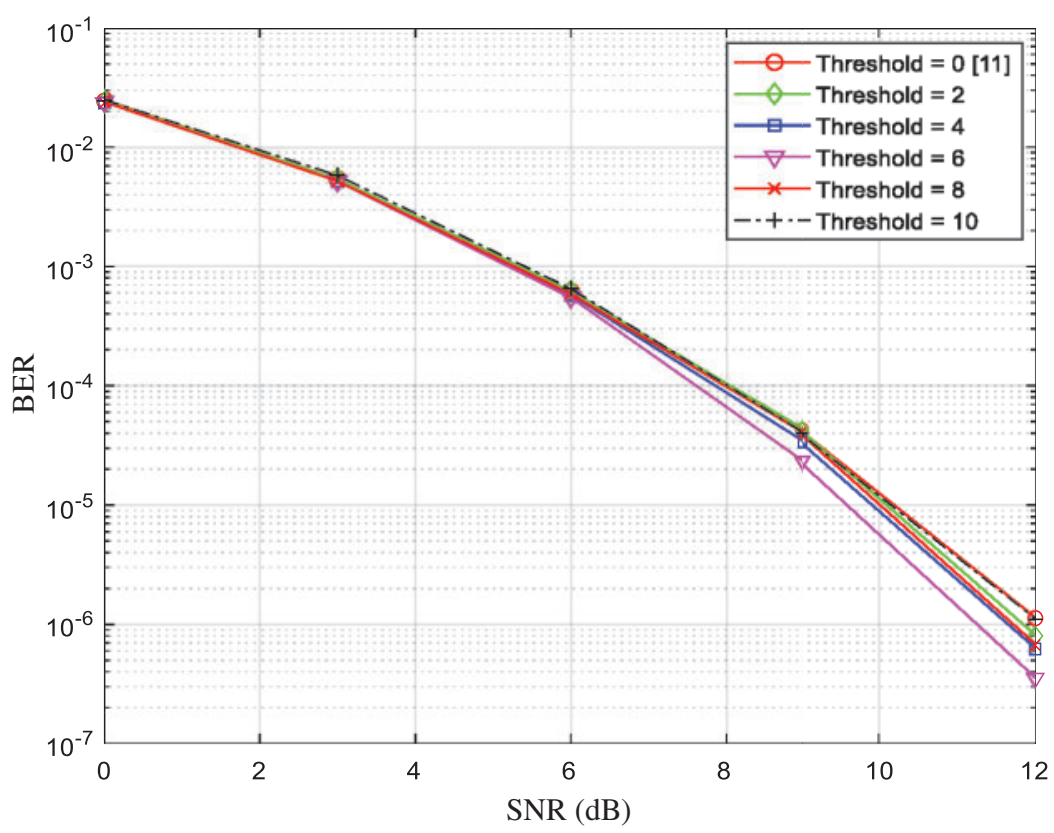

Figure 8: BER vs. SNR for single relay selection strategies using the three-phase relaying protocol with 4-QAM modulation and $\mathcal{R}=7$ 


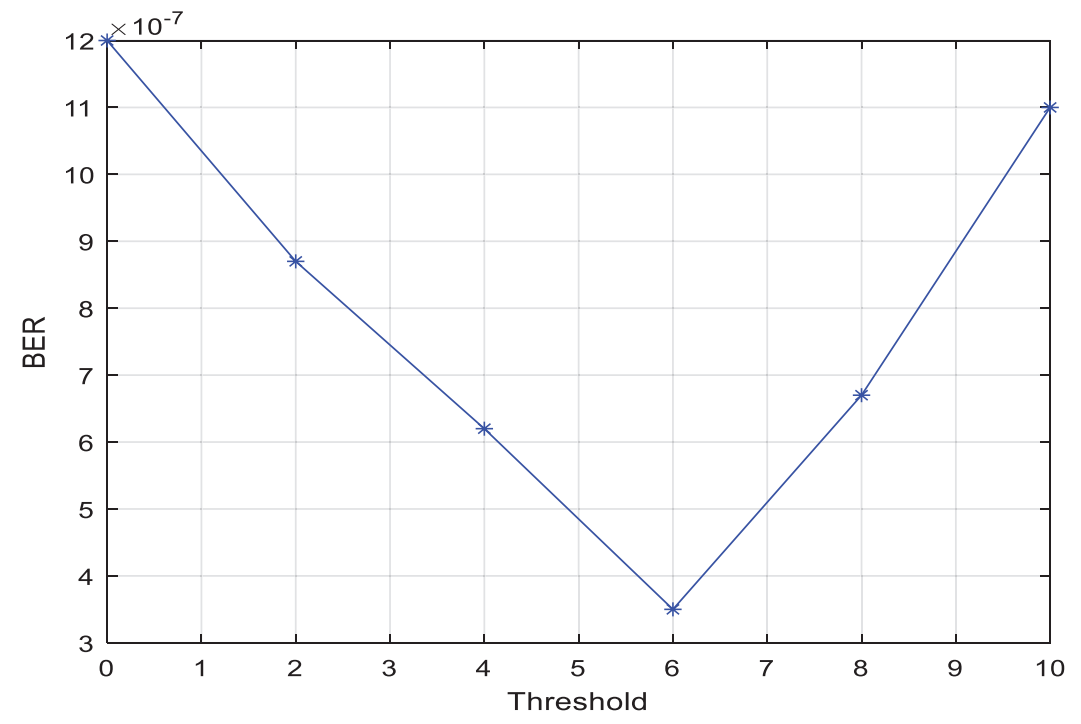

Figure 9: BER vs. Threshold value for single relay selection strategies using the three-phase relaying protocol with $\mathrm{BPSK}$ modulation, $\mathrm{R}=7, \mathrm{SNR}=12 \mathrm{~dB}$

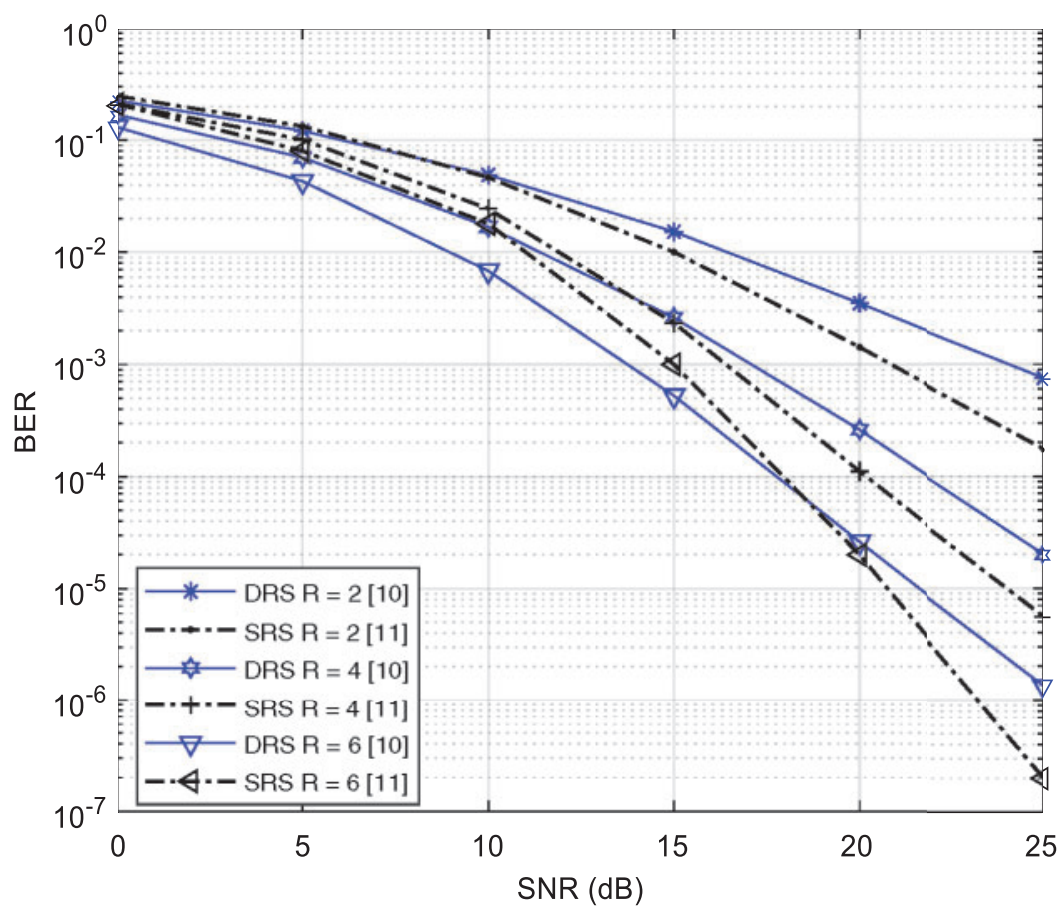

Figure 10: BER vs. SNR for single and dual relay selection strategies using the three-phase relaying protocol with 8 -QAM modulation and $\mathrm{R}=2, \mathrm{R}=4$, and $\mathrm{R}=6$

Fig. 6 clearly shows that our proposed three-phase single-relay-node selection technique under different threshold values outperforms both the three-phase double-relay-node selection technique proposed in [10] and the three-phase single-relay-node selection technique proposed in [11]. Fig. 7 
shows the relation between the BER performance of the relay-node network scenario discussed for Fig. 6 and the threshold values. It is very obvious that this relation is similar to that noticed in Fig. 5, the BER will improve while increasing the threshold value, until the best performance is achieved at threshold value of six (Threshold $=6$ ), then the performance will degrade for threshold values smaller than four. Furthermore, we note here that the BER has been reduced by more than $85 \%$ when we change the threshold value in our proposed technique from zero (standard max-min selection criteria) to six.

Figs. 8 and 9 confirm the results we have achieved and discussed above for the scenarios of four and five relay-nodes network. Also, Fig. 9 shows almost a 70\% reduction in BER when our proposed technique is used compared to the standard max-min selection technique.

Fig. 10 shows that the single-relay selection technique [11] outperforms the double-relay selection technique [10] with increased SNR levels for most of the time. Finally, Fig. 11 clearly shows that our simulation results are highly matching with our theoretical results, obtained from (27) in Section 3, under all scenarios. As explained in Section 4, the optimal threshold value is affected by many factors such as the constellation size, the total transmitted power, and the number of available relay nodes. Therefore, the optimal value depends mainly on the previous three factors. Using Figs. 4-10, we have proved that our proposed single-relay selection technique outperforms the best-known state-of-the-art single-relay selection techniques theoretically and through Monte Carlo simulations. It is also observed that the optimal threshold value varies between four to eight depending on the adopted scenarios. Thus, the optimal threshold value will change if the scenario is changed, i.e., if any of the previous three factors is changed.

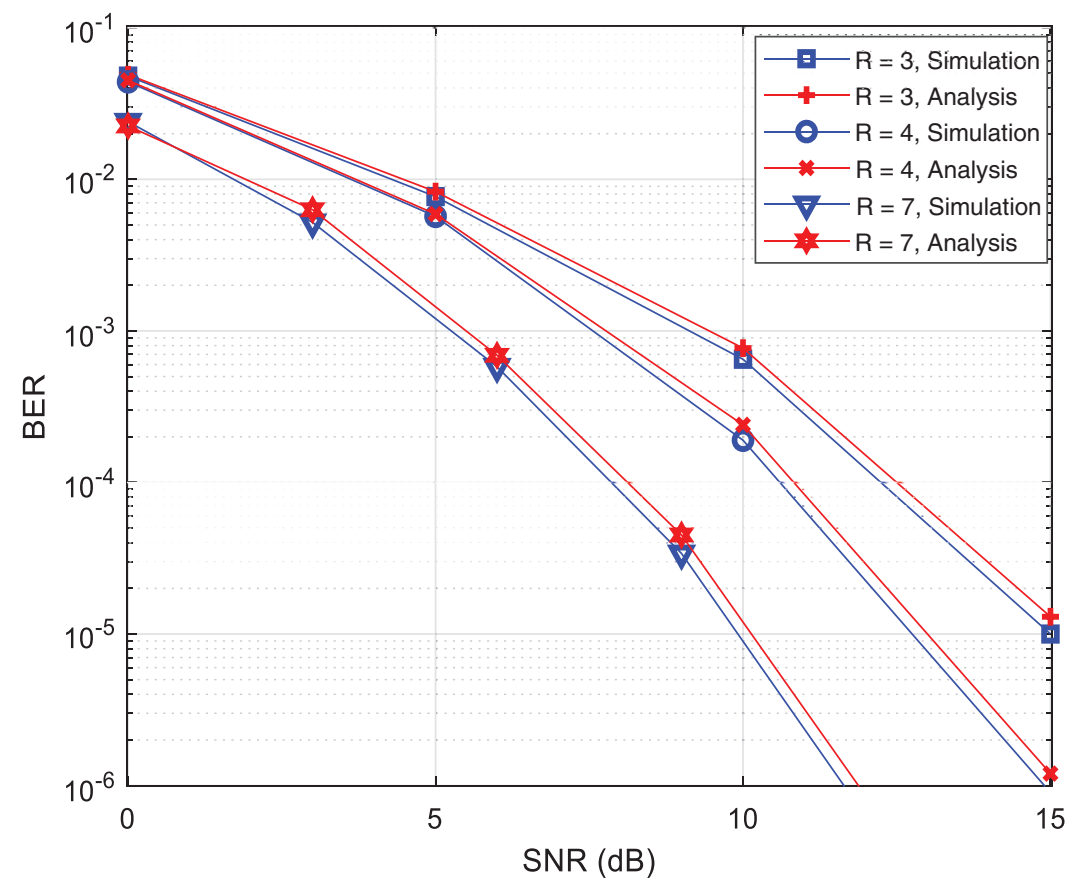

Figure 11: Theoretical and simulated BER performance vs. SNR for the suggested technique with BPSK modulation. $\mathrm{R}=3, \mathrm{R}=4$, and $\mathrm{R}=7$, Threshold $=4$ 


\section{Conclusion}

In this work, we have proposed an improved bi-directional three-phase single-relay-node selection technique for cooperative wireless communications. The new proposed selection technique will select one relay node among $\mathrm{R}$ available relay nodes based on the modified max-min relaynode selection criterion, where in the first step, the best two relay-nodes $\left(\boldsymbol{R}_{\mathrm{a}}\right)$ and $\left(\boldsymbol{\mathcal { R }}_{\mathrm{b}}\right)$ will be selected among the available $\mathrm{R}$ nodes based on the max-min relay-node selection criterion. In the second step we calculate the difference between the minimum link values of the chosen relay-nodes $\left(\mathcal{R}_{\mathrm{a}}\right)$ and $\left(\boldsymbol{\mathcal { R }}_{\mathrm{b}}\right)$. In step three, the best relay-node will be chosen based on a comparison between the calculated difference value and the selected threshold. Also, we have shown through detailed simulations and theoretical analysis that our proposed selection technique outperforms the bestknown state-of-the-art single-relay-node selection techniques, and that our simulation results are almost matching the analytical results.

Funding Statement: This work was supported by College of Engineering and Technology, the American University of the Middle East, Kuwait. Homepage: https://www.aum.edu.kw.

Conflicts of Interest: The authors declare that they have no conflicts of interest to report regarding the present study.

\section{References}

[1] P. Larsson, N. Johansson and K. Sunell, "Coded bi-directional relaying," IEEE Vehicular Technology Conf., vol. 2, pp. 851-855, 2006.

[2] X. Wen, K. Law, S. Alabed and M. Pesavento, "Rank-two beamforming for single-group multicasting networks using OSTBC," in IEEE 7th Sensor Array and Multichannel Signal Processing Workshop. Hoboken, NJ, USA: Stevens Institute of Technology, pp. 65-68, 2012.

[3] D. Taleb, S. Alabed and M. Pesavento, "Optimal general-rank transmit beamforming technique for multicasting service in modern wireless networks using STTC," in Proc. of the 19th Int. IEEE/ITG Workshop on Smart Antennas (WSA 2015), Ilmenau, Germany, 2015.

[4] S. Alabed and M. Pesavento, "A simple distributed differential transmit beamforming technique for two-way wireless relay networks," in The 16th Int. IEEE/ITG Workshop on Smart Antennas (WSA 2012), Dresden, Germany, pp. 243-247, 2012.

[5] A. Schad, S. Alabed, H. Degenhardt and M. Pesavento, "Bi-directional differential beamforming for multi-antenna relaying," in IEEE Int. Conf. on Acoustics, Speech and Signal Processing, South Brisbane, QLD, Australia, pp. 2884-2888, 2015.

[6] S. Alabed, "Performance analysis of differential beamforming in decentralized networks," International Journal of Electrical and Computer Engineering, vol. 8, no. 3, pp. 1692-1700, 2018.

[7] S. Alabed, M. Pesavento and A. Klein, "Non-coherent distributed space-time coding techniques for two-way wireless relay networks," EURASIP Special Issue on Sensor Array Processing, vol. 93, no. 12, pp. 3371-3381, 2013.

[8] S. Alabed, J. Paredes and A. Gershman, "A low complexity decoder for quasi-orthogonal space-time block codes," IEEE Transactions on Wireless Communication, vol. 3, no. 10, pp. 988-994, 2011.

[9] S. Alabed, M. Pesavento and A. Gershman, "Distributed differential space-time coding techniques for two-way wireless relay networks," in Proc. of the Fourth IEEE Int. Workshop on Computational Advances in Multi-Sensor Adaptive Processing, San Juan, Puerto Rico, pp. 221-224, 2011.

[10] Y. Li, R. Louie and B. Vucetic, "Relay selection with network coding in two-way relay channels," IEEE Transactions on Vehicular Technology, vol. 59, no. 9, pp. 4489-4499, 2010.

[11] W. Duan, J. Ju, J. Hou, Q. Sun, X. Jiang et al., "Effective resource utilization schemes for decode-andforward relay networks with NOMA," IEEE Access, vol. 7, pp. 51466-51474, 2019. 
[12] J. Ju, W. Duan, Q. Sun, S. Gao and G. Zhang, "Performance analysis for cooperative NOMA with opportunistic relay selection," IEEE Access, vol. 7, pp. 131488-131500, 2019.

[13] S. Alabed, "Performance analysis of two-way DF relay selection techniques," Information \& Communications Technology Express, Elsevier, vol. 2, no. 3, pp. 91-95, 2016.

[14] G. Cai, Y. Fang, G. Han, J. Xu and G. Chen, "Design and analysis of relay-selection strategies for two-way relay network-coded DCSK systems," IEEE Transactions on Vehicular Technology, vol. 67, no. 2, pp. 1-13, 2017.

[15] T. Nguyen, T. Minh, P. Tran, M. Voznak, T. Duy et al., "Performance enhancement for energy harvesting based two-way relay protocols in wireless ad-hoc networks with partial and full relay selection methods," Ad Hoc Networks, vol. 84, pp. 178-187, 2019.

[16] M. Ju, K. Hwang and H. Song, "Relay selection of cooperative diversity networks with interferencelimited destination," IEEE Transactions on Vehicular Technology, vol. 62, no. 9, pp. 4658-4665, 2013.

[17] Y. Fang, G. Han, P. Chen, F. C. M. Lau, G. Chen et al., "A survey on DCSK-based communication systems and their application to UWB scenarios," IEEE Communications Surveys and Tutorials, vol. 18, no. 3, pp. 1804-1837, 2016.

[18] G. Cai, Y. Fang and G. Han, "Design of an adaptive multiresolution M-ary DCSK system," IEEE Communications Letters, vol. 21, no. 1, pp. 60-63, 2017.

[19] G. Cai, Y. Fang, G. Han, F. C. M. Lau and L. Wang, "A square constellation-based M-ary DCSK communication system," IEEE Access, vol. 4, pp. 6295-6303, 2016.

[20] G. Cai, L. Wang, L. Kong and G. Kaddoum, "SNR estimation for FM DCSK system over multipath Rayleigh fading channels," in IEEE 83rd Vehicular Technology Conf. (VTC Spring), Nanjing, pp. 1-5, 2016.

[21] S. Alabed, M. Pesavento and A. Klein, "Relay selection based space-time coding for two-way wireless relay networks using digital network coding," in Proc. of the 10th Int. Sym. on Wireless Communication Systems (IEEE ISWCS 2013), Ilmenau, Germany, 2013.

[22] S. Alabed, "Performance analysis of bi-directional relay selection strategy for wireless cooperative communications," EURASIP Journal on Wireless Communications and Networking, vol. 1, no. 97, pp. $1-11,2019$.

[23] S. Alabed, "Computationally efficient multi-antenna techniques for multi-user two-way wireless relay networks," International Journal of Electrical and Computer Engineering, vol. 8, no. 3, pp. 1684-1691, 2018.

[24] S. Alabed, "A computationally efficient detector for MIMO systems," International Journal of Electrical and Computer Engineering, vol. 9, no. 5, part II, pp. 4138-4146, 2019.

[25] S. Alabed, J. M. Paredes and A. B. Gershman, "A simple distributed space-time coded strategy for two-way relay channels," IEEE Transactions on Wireless Communications, vol. 11, no. 4, pp. 1260-1265, 2012.

[26] S. Alabed, M. Pesavento and A. Klein, "Distributed differential space-time coding for two-way relay networks using analog network coding," in The 21st European Signal Processing Conf. (EUSIPCO'13), Marrakech, Morocco, 2013.

[27] M. Simon and M. Alouini, Digital communication over fading channels: A unified approach to performance analysis. New York City, United States: John Wiley \& Sons, 2002.

[28] M. Shirzadian Gilan, M. Yavari Manesh and A. Mohammadi, "Cross layer design based on adaptive modulation and queuing for AF cooperative OFDM systems," International Journal of Computer Networks and Wireless Communications, vol. 6, no. 6, pp. 20-28, 2016.

[29] M. Shirzadian Gilan, M. Yavari Manesh and A. Mohammadi, "Level crossing rate and average fade duration of amplify and forward relay channels with co-channel interference," in 22th European Wireless Conf., Oulu, EW, pp. 1-5, 2016. 
[30] M. Y. Manesh and A. Olfat, "Sigmoid function detector in the presence of heavy-tailed noise for multiple antenna cognitive radio networks," in IEEE Int. Conf. on Communications, Paris, pp. 1-5, 2017.

[31] M. Shirzadian Gilan, M. Yavari Manesh and A. Mohammadi, "Optimized target packet error rate for a new cross-layer scheme in AF relay selection system," in IEEE Int. Conf. on Communications, Network, and Satellite, Surabaya, Indonesia, pp. 1-6, 2016. 